\title{
A Linear Programming with Alliance Strategy Coverage in Wireless Sensor Networks
}

\author{
Zeyu Sun ${ }^{12}$, Tao Yang $^{3}$ and Xiaoming Dong ${ }^{4 *}$ \\ ${ }^{1}$ School of Computer and Information Engineering, Luoyang Institute of Science \\ and Technology, Luoyang 471023, China. \\ ${ }^{2}$ Electrical and information Engineering, Xi'an Jiaotong University Xi'an 710049, \\ China \\ ${ }^{3}$ School of Mathematics, Luoyang Institute of Science and Technology, Luoyang \\ 471023, China \\ ${ }^{4}$ School of Information and Technology, Anqing Teachers college, Anqing, Anhui, \\ 246011, China \\ E-mail:dongxiaoming008@126.com
}

\begin{abstract}
How to schedule multiple sensing units of a sensor to cover multiple targets becomes a new challenging problem. This problem is formulated as an integer linear programming problem for maximizing the network lifetime. We propose a novel energy-efficient target coverage algorithm to solve this problem based on clustering architecture. First of all, the network probability model is established and the subordinate relation between sensor nodes and the target nodes is presented. Secondly, a series of probability is computed and the related theorems and reasoning are also proven. Thirdly, effective coverage for the monitoring region is achieved through scheduling mechanism of nodes themselves, thus the purpose of increasing network lifetime can be realized. Finally, experimental results show that the proposed algorithm could achieve complete coverage for networks of different scale, and increase the network lifetime. It possesses the good quality of effectiveness and stability.
\end{abstract}

Keywords: Wireless Sensor Networks; Alliance Strategy; Linear Programming; Coverage rate; Network lifetime

\section{Introduction}

Wireless sensor networks (WSNs) have a wide range of applications, such as military battlefields, environmental monitoring, traffic transportation, medical diagnosis, and undiscovered areas exploring [1]. Usually, a WSN is comprised of many sensor nodes, and one or multiple sink nodes. Each sensor node is composed of a sensor board, a processor, a radio, and a battery to perform sensing, processing, and communication tasks on a covered area. The goals of a WSN are to collect measurements of physical parameters (temperature, humidity, light intensity, etc.) and to detect events in the covered area (wild fire, intrusion, etc.) [2]. WSN compromises the information world and physical world and changes the interactive mode between the nature and human being [13]. At present, WSN has received the attention from all fields and brought immeasurable benefits to the society. Besides, it arouses the research warmness for WSN technology. WSN works in poor environment in which sensor nodes are usually deployed in the monitoring region through randomly spreading [4-5]. Distribution of sensor nodes is very uneven. The energy of it is limited and cannot be recharged. Therefore, it needs considering first to prolong the network life by adopting effective energy-saving coverage method and energy consuming balance mechanism in the research and designing of WSN 
[6-8]. In wireless sensor networks, the ultimate goal of coverage is to allocate each node's state efficiently, minimize the network's energy consumption in each cycle and balance the share of each node's energy consumption in the network without reducing the existing level [9]. The coverage directly reflects the capability of network to monitor the physical world, and energy consumption determines the lifetime of the wireless sensor networks. Network coverage and energy consumption are closely related.

The structure of hybrids sensor network is almost identical with WSN, but a certain amount of mobile sensor nodes with mobility property makes the cost slightly higher than traditional WSN. However, the number of mobile nodes can be set according to specific application, and the cost can be limited in the affordable range. Due to the merits of hybrid sensor network, the related researches are more than other researches. More often than not, hybrid sensor networks are studied in the research of WSN, such as coverage and connectivity, location, routing, and data fusion, etc. All the problems in the networks can be solved in better ways in hybrid sensor networks by using the mobile nodes, such as coverage. If mobile nodes in mobile sensor network are relocated, the nodes can be distributed more evenly and the coverage hole can be filled. By this way, the quality of coverage in the network can be improved.

\section{Related Works}

For the coverage optimization problem, Wang et al. [9] proposed a linear programming-based approximation algorithm to minimize the deployment cost in HWSNs, where the sensors are with different sensing ranges and costs. The purpose of this algorithm was that each target is required to be covered by at least $\mathrm{k}$ sensors, and the total cost of the sensors is minimized. However, this algorithm only considered the situation that a sensor just has one sensing unit. Capone et al. [10] considered multi-hop coverage and connectivity between sensor nodes and gateway nodes. An optimizing framework based on integer linear programming (ILP) was developed to locate wireless gateways while minimizing the installation cost and energy consumption. A heuristic algorithm was also proposed for large-scale heterogeneous network scenarios, and the quality of the designed networks is evaluated. For maximizing coverage time of clustered WSNs, Shu et al. [11] presented a coverage-time-optimal algorithm, in which the optimal clustering and routing are computed using linear programming. The maximizing coverage-time problem is solvable in linear time under stochastic setups. The deployment of information coverage and random coverage was conducted by using the sensor density relation, and the upper bound value of probability was given to calculate that of information coverage and the events [13]. The stochastic scheduling algorithm was studied, including simulation of network coverage intensity, delay detection and probability of detection. Bounded detection delay, detection probability, and network coverage intensity with constraints of network life cycle QOS [14]. A distribution method was proposed to provide complete coverage performance with the help of random scheduling, including: random partitions and coverage improvement. The Voronoi polygon method and the disk coverage calculation rule were used [15]. For the WSN with intensively-distributed nodes, a coverage control protocol named "Node Self-Scheduling" was proposed. Nodes in the network were firstly divided into many disjoint sets to ensure complete coverage of the target area, then these sets of nodes shifted their state between "active" and "sleeping".[16].Berman improved Slijepcevic's thought. He did not require disjoint of nodes sets, allowing each nodes set work for a certain time. Besides, the partial coverage was also considered. In this way, more node sets could be divided and the network life time could be prolonged [17]. Tian improved Slijepcevic with back-off mechanism and the network lifetime could be effectively prolonged. Emergence of network coverage hole could be avoided. Redundant nodes could be reduced. The network could be fully covered and a certain sensing reliability could be maintained. 
However, this rotation mechanism only applied to nodes of two dimensional perceptual models. It was not suitable for nodes in the probability perceptual model. And there would be some additional costs for the network, such as the requirement of knowledge of node position and synchronization the network time [18]. A coverage algorithm based on Graph coloring strategy, which aimed at finding the maximum disjoint advantage set, could effectively prolong the lifetime of WSN, but it required that all the nodes in the set must not be disabled[19]. In the covering process, the requirements for target nodes' coverage were different. Based on this, a network configuration protocol (CCP) was proposed, which minimized the number of working nodes, with the precondition of ensuring the network coverage rate. Two greedy algorithms: centralized and distributed, were also proposed respectively by combining CCP and SPAN in order to ensure network coverage and connectivity [20]. From the perspective of border coverage, a centralized and polynomial time detection algorithm was proposed. It adapted to the environment in which positioning and monitoring capability or fault tolerance was highly required [21]. As for target coverage, what we should resolve is $m$ targets of known position were given and $\mathrm{n}$ monitoring nodes were deployed. Due to the limited energy of every sensor node, it was a problem that how to deploy nodes in a reasonable range and how to maximize the network lifetime on the basis of target monitoring. Cardei put forward a heuristic algorithm by using mixed integer programming [22]. Then he discussed the coverage problem in the case of node sets intersection and adjustable sensing radius of node. When the targets' density was high, Cardei approximated the target coverage as region coverage and realized the connectivity coverage of the target through establishing a connected set of nodes with the help of sensing nodes in high density. Based on the studies of Cardei, Liu and other researchers further studied the target coverage problem. He constrained one node to cover only one target each time [23]. It could be ensured that target coverage problem could be resolved effectively in polynomial time. Wu proposed a protocol which constructed distributed localization of connected dominating set and discussed methods of dominating set coverage with efficient energy. To balance the network energy consumption, a method of selection coverage nodes was presented through remaining energy level [24]. Through the above methods, only static nodes were used to cover the target continuously, but in some applications, it was not necessary to provide continuous coverage of target. It would be much more efficient to use some of mobile nodes than just use static nodes. A polynomial approximation algorithm was presented by constructing a minimum spanning tree [25]. It was also discussed how to reduce the number of nodes and how to determine the position of nodes to cover a given target in the secure and controllable network environment. In addition, the connectivity of network must be ensured.

\section{Basic Problem and Network Model}

For the convenience of study, the algorithm in this paper is based on the following four assumptions:

(1)The communications range and sensing range of wireless sensor nodes are discshaped. The position of each node can be obtained through some positioning algorithm.

(2)The nodes in the WSN are morphology and independently; each node has the same sensing range but different communications range.

(3)All the nodes in WSN are randomly deployed in a square area. The border factor and the condition in which the border exists should also be considered.

(4)At first, each node has the same energy and they are in the same status in the network. 


\subsection{Basic Definitions}

Definition 1: The fact some area is covered by the node $s_{i}$ means any point $p$ in this area is within the coverage of $s_{i}$. That is: $A s_{i}=\left\{p \mid d(p,(x, y)) \leq r_{s}, i \in[1, n]\right\} . d$ is the Euclidean distance between two points. $r_{s}$ is the sensing radius of nodes. $n$ is the number of nodes.

Definition 2: The coverage rate of in WSN $W$ deployed in target are $\Omega$, is $C_{i}(W, \Omega)=\int_{\Omega} s\left(x_{i}\right) d x / S(\Omega) . S(\Omega)$ is the area of the target region, when $D\left(s_{i}, t_{k}\right) \leq 1$, $S(\Omega)=1$, otherwise, $S(\Omega)=0$.

Definition 3: There exists node $s_{i}$ and $s_{j}$. The target regions they covered are $\Omega_{i}$ and $\Omega_{j}$ respectively. if $\Omega_{i} \cap \Omega_{j} \neq \varnothing$, then node $s_{i}$ and $s_{j}$ is regarded to be coverage related.

\subsection{Network Model}

For sake of conveniences, the sensor nodes and the target nodes are placed in a square region. Generally, the coverage level of the target directly reflects the level that the target node is concerned. The target node region being concerned has a higher coverage level. The function of expectation value of different areas where the sensor node $\mathrm{P}$ is located and the coverage area should be taken in consideration, as is shown in Figure 1:

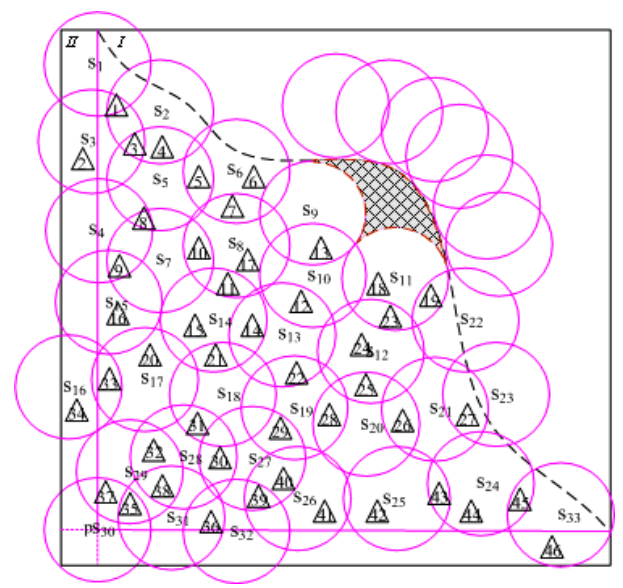

Figure 1. Schematic for Associated Coverage Attributes

Figure 1 shows the associated coverage relationship of sensor nodes and target nodes. Circles represent the sensor nodes; Triangle represents the target nodes; Dotted lines in the figure represent moving locus of mobile targets; in the lower left corner, node P is defined; Shadows are blind regions which are not covered by sensor nodes. When the target node moves from the upper left to lower right of the figure to the sensor nodes 9 and 11, some region cannot be covered. We call it empty or blind area. In Figure 1, four problems are studied as follows:

(1)What's the relation of the associated attributes between wireless sensor nodes and target nodes?

(2) For target nodes, how to denote the relationship of wireless sensor nodes and the target nodes?

(3) What is the relationship between the area of blind region and that of wireless sensor nodes?

(4) How to compute the coverage level, density functions and the expectation value of the nodes in WSN?

(5)How to determine the coverage of target area with the least deployed nodes by using the probability expectation value? How to realize scheduling mechanism of sensor nodes? 
Let's study the first problem: what's the relationship of the associated attributes between wireless sensor nodes and target nodes? In Figure 1, every sensor node covers one or more target nodes except the part above the dotted lines. That means some of the target nodes are covered by more than one sensor nodes, namely, $\mathrm{K}$ degree Coverage. A chart is used to represent the associated attributes between WSN nodes and the target nodes. As is shown in Table 1:

\section{Table 1. The Associated Attributes between Wireless Sensor Nodes and Target Nodes}

\begin{tabular}{cccccc}
\hline $\begin{array}{c}\text { Associated } \\
\text { sensor nodes }\end{array}$ & $\begin{array}{c}\text { Associated } \\
\text { target nodes }\end{array}$ & $\begin{array}{c}\text { Associated } \\
\text { sensor nodes }\end{array}$ & $\begin{array}{c}\text { Associated } \\
\text { target nodes }\end{array}$ & $\begin{array}{c}\text { Associated } \\
\text { sensor nodes }\end{array}$ & $\begin{array}{c}\text { Associated } \\
\text { target nodes }\end{array}$ \\
\hline $\mathrm{S}_{1}$ & 1 & $\mathrm{~S}_{12}$ & 23 & $\mathrm{~S}_{23}$ & 27 \\
$\mathrm{~S}_{2}$ & $1,3,4$ & $\mathrm{~S}_{13}$ & $12,14,22$ & $\mathrm{~S}_{24}$ & $43,44,45$ \\
$\mathrm{~S}_{3}$ & $1,2,3$ & $\mathrm{~S}_{14}$ & $11,14,15,21$ & $\mathrm{~S}_{25}$ & 42,43 \\
$\mathrm{~S}_{4}$ & 8,9 & $\mathrm{~S}_{15}$ & 9,16 & $\mathrm{~S}_{26}$ & $39,40,41$ \\
$\mathrm{~S}_{5}$ & $3,4,5,8$ & $\mathrm{~S}_{16}$ & 33,34 & $\mathrm{~S}_{27}$ & $29,30,39,40$ \\
$\mathrm{~S}_{6}$ & $5,6,7$ & $\mathrm{~S}_{17}$ & 20,33 & $\mathrm{~S}_{28}$ & $30,31,32,38$ \\
$\mathrm{~S}_{7}$ & $8,9,10$ & $\mathrm{~S}_{18}$ & 21,31 & $\mathrm{~S}_{29}$ & $32,35,37,38$ \\
$\mathrm{~S}_{8}$ & $7,10,11,17$ & $\mathrm{~S}_{19}$ & $22,28,29$ & $\mathrm{~S}_{30}$ & 35,37 \\
$\mathrm{~S}_{9}$ & 13 & $\mathrm{~S}_{20}$ & $25,26,28$ & $\mathrm{~S}_{31}$ & $35,36,38$ \\
$\mathrm{~S}_{10}$ & 12,13 & $\mathrm{~S}_{21}$ & 26,27 & $\mathrm{~S}_{32}$ & 36,39 \\
$\mathrm{~S}_{11}$ & $18,19,23$ & $\mathrm{~S}_{22}$ & 19 & $\mathrm{~S}_{33}$ & 45,46 \\
\hline
\end{tabular}

From table 1, we can see that each target node is covered by one or more sensors, and the coverage degree for each target node is not the same. When the target node is covered by multiple sensor nodes, it may be the main target node being concerned, but if it is covered by multiple sensor nodes $(K \geqq 2)$, it may not be the one being concerned. Some sensor nodes can be put into a dormant state in order that the energy consumption of the sensor nodes can be reduced. This will be further explained in the subsequent chapters.

The second problem is exactly the inverse problem of the first one. That is, a target node is covered by multiple sensors and the K-degree coverage is formed. The formed association relationship between this target node and sensor nodes will be studied in the second question. The association relationship between target nodes and sensor nodes is shown in Table 2:

Table 2. The Associate Attributes between Wireless Sensor Nodes and Target Nodes

\begin{tabular}{|c|c|c|c|c|c|}
\hline $\begin{array}{l}\text { Associated } \\
\text { target node }\end{array}$ & $\begin{array}{l}\text { Associated } \\
\text { sensor node }\end{array}$ & $\begin{array}{l}\text { Associated } \\
\text { target node }\end{array}$ & $\begin{array}{l}\text { Associated } \\
\text { sensor node }\end{array}$ & $\begin{array}{l}\text { Associated } \\
\text { target node }\end{array}$ & $\begin{array}{l}\text { Associated } \\
\text { sensor node }\end{array}$ \\
\hline 1 & $\mathrm{~S}_{1}, \mathrm{~S}_{2}, \mathrm{~S}_{3}$ & 16 & $\mathrm{~S}_{15}$ & 31 & $\mathrm{~S}_{18,} \mathrm{~S}_{28}$ \\
\hline 2 & $\mathrm{~S}_{3}$ & 17 & $\mathrm{~S}_{8}$ & 32 & $\mathrm{~S}_{28,}, \mathrm{~S}_{29}$ \\
\hline 3 & $\mathrm{~S}_{2}, \mathrm{~S}_{3}, \mathrm{~S}_{5}$ & 18 & $\mathrm{~S}_{11}$ & 33 & $\mathrm{~S}_{6}, \mathrm{~S}_{17}$ \\
\hline 4 & $\mathrm{~S}_{2}, \mathrm{~S}_{5}$ & 19 & $S_{11}, S_{22}$ & 34 & $\mathrm{~S}_{16}$ \\
\hline 5 & $\mathrm{~S}_{5}, \mathrm{~S}_{6}$ & 20 & $\mathrm{~S}_{17}$ & 35 & $\mathrm{~S}_{29}, \mathrm{~S}_{30}, \mathrm{~S}_{31}$ \\
\hline 6 & $\mathrm{~S}_{6}$ & 21 & $\mathrm{~S}_{14,} \mathrm{~S}_{18}$ & 36 & $\mathrm{~S}_{31}, \mathrm{~S}_{32}$ \\
\hline 7 & $\mathrm{~S}_{6}, \mathrm{~S}_{8}$ & 22 & $\mathrm{~S}_{13}, \mathrm{~S}_{19}$ & 37 & $\mathrm{~S}_{29}, \mathrm{~S}_{30}$ \\
\hline 8 & $\mathrm{~S}_{4}, \mathrm{~S}_{5}, \mathrm{~S}_{7}$ & 23 & $\mathrm{~S}_{11}, \mathrm{~S}_{12}$ & 38 & $\mathrm{~S}_{28}, \mathrm{~S}_{29}, \mathrm{~S}_{31}$ \\
\hline 9 & $\mathrm{~S}_{4}, \mathrm{~S}_{7}, \mathrm{~S}_{15}$ & 24 & $\mathrm{~S}_{12}$ & 39 & $\mathrm{~S}_{26,} \mathrm{~S}_{27}, \mathrm{~S}_{32}$ \\
\hline 10 & $\mathrm{~S}_{7}, \mathrm{~S}_{8}$ & 25 & $\mathrm{~S}_{12,} \mathrm{~S}_{20}$ & 40 & $\mathrm{~S}_{26}, \mathrm{~S}_{27}$ \\
\hline 11 & $\mathrm{~S}_{8,}, \mathrm{~S}_{14}$ & 26 & $\mathrm{~S}_{20,} \mathrm{~S}_{21}$ & 41 & $\mathrm{~S}_{26}$ \\
\hline 12 & $S_{10}, S_{13}$ & 27 & $S_{21}, S_{23}$ & 42 & $\mathrm{~S}_{25}$ \\
\hline 13 & $\mathrm{~S}_{9,} \mathrm{~S}_{10}$ & 28 & $\mathrm{~S}_{19,} \mathrm{~S}_{20}$ & 43 & $\mathrm{~S}_{24}, \mathrm{~S}_{25}$ \\
\hline 14 & $\mathrm{~S}_{13}, \mathrm{~S}_{14}$ & 29 & $\mathrm{~S}_{19}, \mathrm{~S}_{27}$ & 44 & $\mathrm{~S}_{24}$ \\
\hline 15 & $\mathrm{~S}_{14}$ & 30 & $S_{27}, S_{28}$ & 45 & $\mathrm{~S}_{24}, \mathrm{~S}_{33}$ \\
\hline
\end{tabular}


From Table 2, we can see that each target node is covered by one or more sensors and the coverage degree for each target node is not the same. When a target node is covered by multiple sensor nodes, multiple covering is formed. For example: target node 8 is in the coverage range of S4, S5, S7 and the degree of the coverage is 3 . If the coverage degree for a target node is higher than needed, there must existed many redundant nodes. This will consume much energy of the entire network and the network lifetime will be decreased. With the increasing of sensor nodes, more than one target nodes are most likely to be in the multiple coverage area. Large sum of redundant nodes will be generated with the precondition that a certain coverage degree can be realized. In order to avoid the existence of many redundant nodes, all the sensor nodes should be put in different states and the transition of different states should be accomplished.

\subsection{The Improved Coverage Algorithm}

Theorem 1. Given the side length of a square region 1, the sensing radius of the node $r_{s}$, and a parameter, in order to ensure that the expected network coverage ratio is not less than, the number of randomly deployed nodes is at least

$$
\ln (1-\varepsilon) / \ln (1-\mathrm{E}(S) / \Omega)
$$

Proof: According to formula (1), $P\left(S_{n}\right)=l-(l-E[C] / \Omega)^{n} \geq \varepsilon$, then the result can be get: $n \ln (1-E[C] / \Omega) \leq \ln (1-\varepsilon)$. Because $\ln (1-E[C] / \Omega)<0, n \geq \ln (1-\varepsilon) / \ln (1-E[C] / \Omega)$.

Theorem 2: Without loss of generality, if the holes exist, i.e. $x \geq 0$, and is continuously differentiable in the range of $[0, a]$.Besides, if the track equation of the hole satisfies $f_{n}(x)=\int_{0}^{x} f_{n-1}(x) d x$, then progression $\sum_{n=0}^{\infty} f_{n}(x)$ must be absolute convergence in the range of $[0, a]$.

Proof: Because $x \geq 0$ and it is continuously differentiable in $[0, a],\left|f_{0}(x)\right|$ is continuous in $[0, a]$ and there is the maximum value of $\left|f_{0}(x)\right|$ in the range of $[0, a]$. Let $\mathrm{M}$ be the maximum value, $M=\max _{0 \leq x \leq a}\left|f_{0}(x)\right|$. Because:

$$
\begin{gathered}
f_{n}(x)=\int_{0}^{x} f_{n-1}(x) d x=\int_{0}^{x}\left[\int_{0}^{x} f_{n-2}(x) d x\right] d x= \\
\quad \cdots=\int_{0}^{x} \cdots \int_{0}^{x} f_{0}(x)(d x)^{n}
\end{gathered}
$$

Because $\left|f_{0}(x)\right|$ is continuous in $[0, a]$, there exists the maximum value- $\mathbf{M}$ of $\left|f_{0}(x)\right|$ in $[0, a]$.Consequently:

$$
\left|f_{n}(x)\right| \leq \int_{0}^{x} \cdots \int_{0}^{x}\left|f_{0}(x)\right| d x \leq \int_{0}^{x} \cdots \int_{0}^{x} M d x=\frac{M}{n !} x^{n}
$$

Because Formula (9) is convergent when $x \in[0, a], \sum_{n=0}^{\infty}\left|f_{n}(x)\right|$ is convergent. i.e. $\sum_{n=0}^{\infty} f_{n}(x)$ is absolute convergent.

In the following sections, the relationship between the coverage area and its expectation value is analyzed through the example of sensor node 30(node p) in the lower left corner.

A is shown in Figure 1; the square region 1 is divided into two parts: region 1 and region 2 . The nodes are randomly deployed in the monitoring region and constituted in a limited set $\mathrm{S}$. The coverage area of each node is $\mathrm{E}(C)$. The coverage probability of each node is $\mathrm{E}(\mathrm{C}) / \Omega$. If $\mathrm{S}$ is empty, the coverage rate of the deployed n nodes is $\mathrm{P}(\mathrm{S})=(1-$ $\mathrm{E}(C) / \Omega)^{\mathrm{n}}$. When set $\mathrm{S}$ is not empty, the value of coverage probability of network nodes is as below:

$$
P(S)=1-(1-E(C) / \Omega)^{n}
$$


When node number $n \rightarrow \infty, \lim _{n \rightarrow \infty} E(P(S))=1$. This means that when the number of nodes is large enough, the monitoring region will be fully covered. Considering the boundary effect, the node coverage area and its expectation value is to be solved. Because the square region is divided into regions 1 and 2, according to the definition of expectation value in probability theory, the expectation value of nodes coverage area in the network can be conducted as follows:

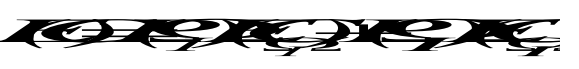

$P\left(\Omega_{I}\right)$ and $P\left(\Omega_{I I}\right)$ denote the probability of the node randomly deployed in region 1 and region 2 respectively. $E\left(C_{\Omega_{I}}\right)$ and $E\left(C_{\Omega_{I}}\right)$ represents the corresponding coverage expectation respectively. Because the deployment of sensor nodes follows uniform distribution, thereby the following result is obtained:

$$
\left\{\begin{array}{l}
P\left(\Omega_{T}\right)=\left(l-2 r_{s}\right)^{2} / l^{2} \\
R\left(\Omega_{\pi}\right)=4 r_{s}\left(l-r_{s}\right) / l^{2}
\end{array}\right.
$$

Assuming node $\mathrm{p}$ is inside region 1 , its coverage range is completely contained, so the coverage expectation is:

$$
\boldsymbol{H}\left(\boldsymbol{C}_{\Omega}\right)=\pi_{\mathrm{s}}^{2}
$$

When node $p$ is inside region2, the area is that of its sensing circle minus that of arch region $S_{\mathrm{ACBD}}$. A and B is the intersection of the sensing circle of node $\mathrm{p}$ and the network border. Its angle $\theta$ is the central angle formed by node $\mathrm{p}$, point $\mathrm{A}$ and point $\mathrm{B}$, i.e. $\angle$ $\mathrm{ApB}=\theta$. The central angle $\theta=2 \arccos y / r_{s}$, then formula (14) is obtained:

$$
\begin{aligned}
E\left(C_{\Omega_{I I}}\right) & =4 \int_{0}^{l-r_{s}} \frac{1}{2} r_{s}^{2}(2 \pi-\theta+\sin \theta) d x \int_{0}^{r_{s}} \frac{1}{4\left(l-r_{s}\right) r_{s}} d y \\
& =\frac{r_{s}}{2\left(l-r_{s}\right)} \int_{0}^{l-r_{s}}(2 \pi-\theta+\sin \theta) d x \int_{0}^{r_{s}} d y
\end{aligned}
$$

Theorem 3: Supposing that given sensor nodes with sensing radius rs, is uniformly distributed in the square region with side length of 1 , considering the boundary factors, the coverage expectation of each node is:

$$
E(C)=\left(\frac{r_{s}}{l}\right)^{2}\left[\pi\left(l-2 r_{s}\right)^{2}+2\left(l-r_{s}\right)\left(2 \pi r_{s}+\frac{\pi}{2}+r_{s}\right)\right]
$$

Proof: Because the sensor nodes follow uniform distribution, from formula (6), the coverage expectation is: $E(C)=P\left(\Omega_{I}\right) E\left(C_{\Omega_{I}}\right)+P\left(\Omega_{I I}\right) E\left(C_{\Omega_{I}}\right)$. When formula (7), (8), (9) are put into formula (9), the result is:

$$
\begin{aligned}
E(C)= & P\left(\Omega_{I}\right) E\left(C_{\Omega_{l}}\right)+P\left(\Omega_{I I}\right) E\left(C_{\Omega_{I l}}\right) \\
= & \frac{1}{l^{2}}\left[\pi r_{s}^{2}\left(l-2 r_{s}\right)^{2}\right]+\frac{1}{l^{2}}\left[2 r_{s}^{2}\right. \\
& \left.\int_{0}^{l-r_{s}}(2 \pi-\theta+\sin \theta) d x \int_{0}^{r_{s}} d y\right] \\
= & \left(\frac{r_{s}}{l}\right)^{2}\left[\pi\left(l-2 r_{s}\right)^{2}+2\left(l-r_{s}\right)\left(2 \pi r_{s}+\frac{\pi}{2}+r_{s}\right)\right]
\end{aligned}
$$

\section{Evaluation of Performance}

In order to study this subject better, the meaning of each parameter is listed one by one:

$l$ : the side length of a square

$\Omega$ : the area for a square, i.e.

$n$ : the number of randomly deployed sensor nodes

$r$ : the sensing radius of sensor nodes

$\mathrm{E}(C)$ : the expectation of coverage area of sensor nodes, i.e. 
$\sigma^{2}:$ the variance of coverage area of sensor nodes

$p(x)$ : the coverage rate of randomly deployed sensor nodes

In order to improve the evaluation of network performance, MATLAB6.5 is used in simulation experiment. Coverage and connectivity of network in different scales can be realized through changing range of the coverage region. The model performance in different scales can be evaluated better. It is mainly reflected in the minimum number of nodes deployed in the cases of different coverage and network connectivity rate. The average value is derived from simulations of 100 times. The curve for node coverage variation in different network scales is shown in Figure 2:

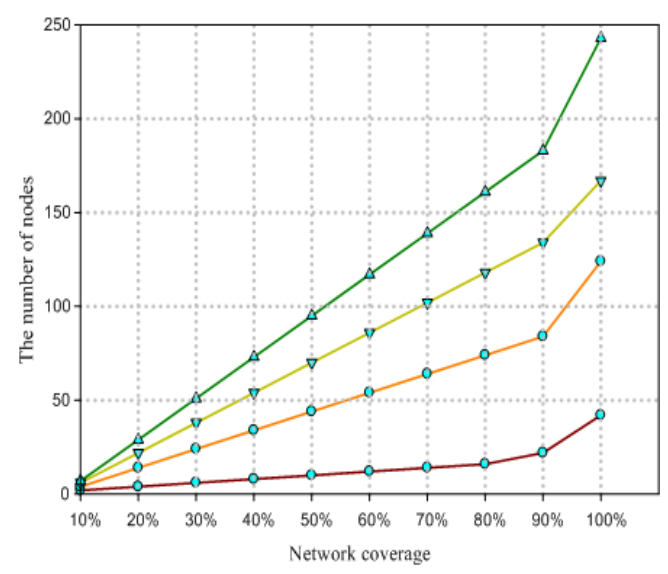

Figure 2. Curve for Node Coverage Variation in Different Network Scales

As can be seen in the Figure 2, firstly, there are fewer nodes for complete coverage in the network with smaller area and more nodes in larger area. For example, when coverage is $100 \%$, 43 nodes are needed in $100 * 100$ area; 124 nodes in $200 * 200$ area; 167 nodes in $300 * 300$ area; 243 nodes in $400 * 400$ area. Secondly, the number of sensor nodes is not the same according to different requirements of networks for coverage rate. The number of nodes is increasing as the network grows, which shows a linear increasing relationship. In Figure 2, it requires more nodes to realize full coverage for much larger networks and fewer nodes for smaller networks. The coverage rate is between $90 \%$ and $100 \%$, the increment rate of large network increases faster than that of small-scale networks.

To further verify the coverage rate of WSN in probability model, the $100 * 100$ model is chosen for study. After the network parameters are given dynamically, the proportion between network coverage rate and the number of nodes is compared, as is shown in Figure 3

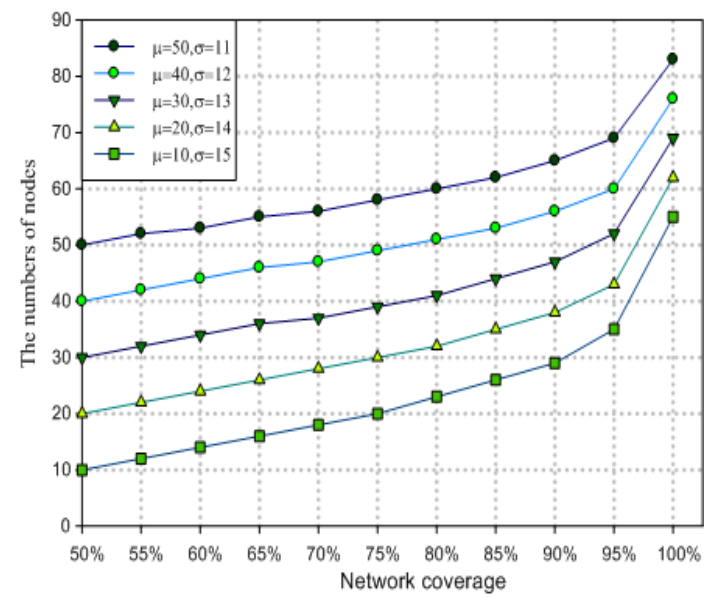

Figure 3. Curve for Network Coverage Variation with Different Parameters 
Figure 3 shows the network coverage rate under different parameters, its computation process is according to formula (7), when stochastic variable follows normal distribution, is used to get the result. Take as an example, when the coverage rate is $60 \%$, it can be deducted that, and when the coverage rate is $60 \%$, the required number of nodes is 14 . From Figure 3, we can also see that, in case of same network size, the larger the expectation, the more nodes will be required, i.e. The normal distribution model that the network shows. For any curve, the variation is linear relationships that is because, the first: the coverage rate increase at $5 \%$, so the distributed function values changes slowly. Second, the difference of expectation in the example is 10, which means the two adjacent curves have the feature of equal spacing. Third, when the coverage rate exceeds $95 \%$, the number of nodes increased significantly. That is due to the quick change of distributed function values between $95 \%$ and $99.9 \%$. For example, if the expectation is 10 and variance is 15,35 nodes are needed with coverage rate $95 \%$ while 55 is needed with coverage rate $99.9 \%$.

For another important factor of WSN connectivity, the size of connectivity is directly related to the performance of data transmission, data processing and data computation, etc. Next four different network models are adopted to compare the connectivity of WSN in experiments. According to formula (8), (9), and (10), the number of sensor nodes in different network models is solved, as is shown in Figure 4:

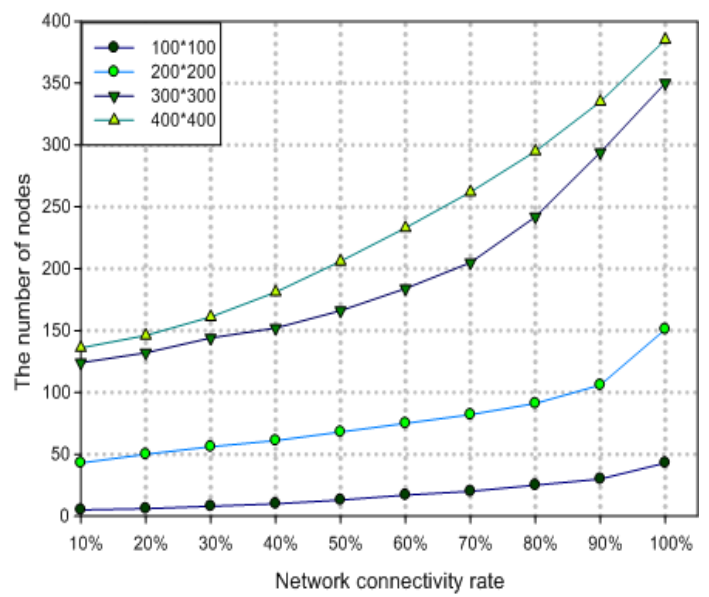

Figure 4. Curve for Network Connectivity in Different Network Models

Figure 4 reflects the variation curves between network connectivity rate and the number of nodes. For the two networks of $100 * 100$ and $200 * 200$, the number of nodes increases more slowly. The main reasons are: First, when the network is small and the connectivity rate is $100 \%, 48$ nodes can complete the connectivity between nodes in the network model of $100 * 100$ while 150 nodes are needed in the network model of $200 *$ 200. Second, for the two network models, the increasing trends of the nodes number are relatively stable. The node number increments are in linear relationship with time. For the two networks of $300 * 300$ and $400 * 400$, because their areas are larger, more nodes are needed compared with the previous two network models. At the beginning, the nodes number in the two network models are almost the same. However, with the expansion of the network model, the nodes are greatly increasing in $400 * 400$ network model, because in the communication process, the optimal communication rule is the two circles of sensor nodes are circumscribed, which is, however, impossible to achieve. Therefore, as the network grows, much more nodes are largely required. 


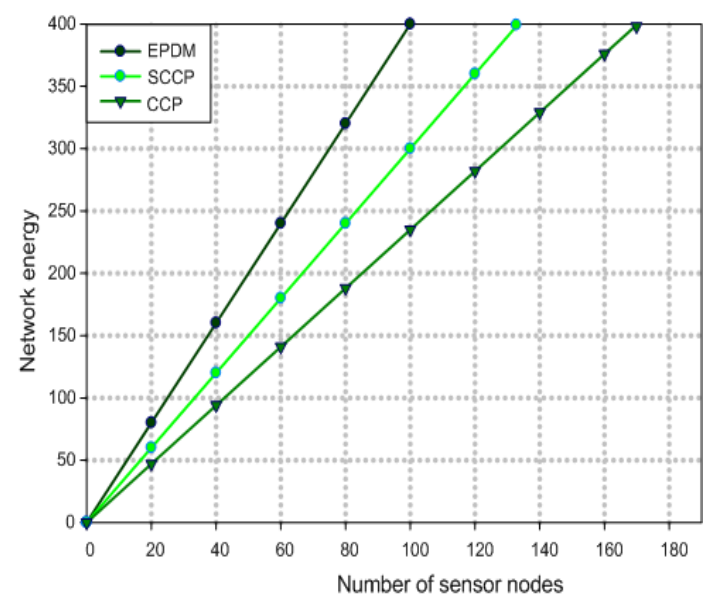

Figure 5. The Relationship between Number of Sensor Nodes and Network Energy

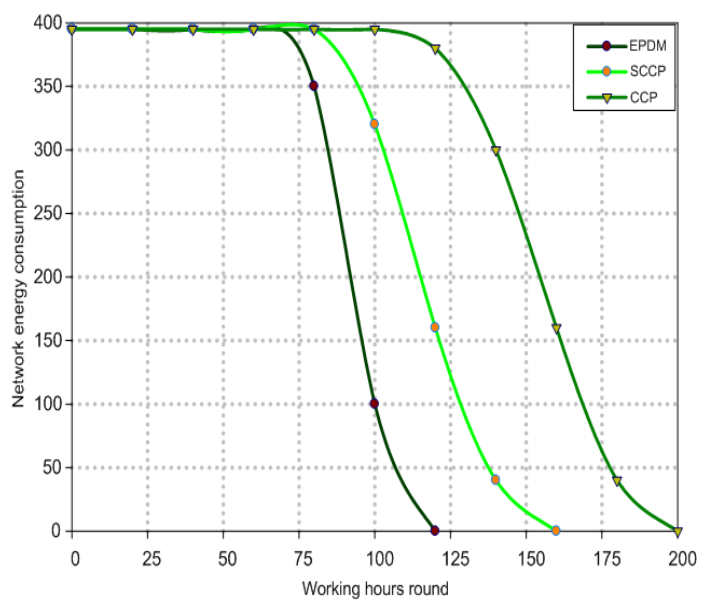

Figure 6. Curve for Node Energy and Time Rounds

Figure 5 and Figure 6 shows the comparison between network energy and nodes number along with the rest energy of nodes changing with time for the paper algorithm and algorithms of CCP and SCCP with the precondition of network coverage. As can be seen from the figure, with the same nodes number, the progressively increased speed of the paper algorithm is larger than that of the algorithms of CCP and SCCP. For a certain energy value, the nodes of the paper algorithm are much less than that of the other two algorithms. Figure 6 reflects the total remaining energy of network nodes is decreased gradually with time in the operation of system. Compared with the other two algorithms, less energy is consumed while the network coverage can be ensured through this algorithm. After the network runs for the same period of time, 9\% energy in average can be saved by using this algorithm than SCCP and 17\% energy than CCP. This is because it costs little in the calculation of network coverage and less energy of sensor nodes is consumed.

\section{Conclusions}

This paper researches the coverage algorithm in wireless sensor networks and presents the paper algorithm with the theory of probability. First, the paper establishes the relation model and association relation between sensor nodes and target nodes. Then, the calculation of a target node's trajectory is given based on its probability and expectation. Next, target nodes can be covered more effectively through scheduling mechanism of 
nodes. The simulation results show the paper is effective and scalable. In future research, the paper will be extended to implement the multiple coverage is in heterogeneous wireless sensor network. Furthermore, we will study how to do quadratic linear programming for nodes with random distribution and improve the calculating precision for the border coverage.

\section{Acknowledgments}

Projects (61170245,U1304603) supported by the National Natural Science Foundation of China; Project (2014B520099) supported by Henan Province Education Department Natural Science Foundation; Project (142102210471,142102210063, 142102210568) supported by Natural Science and Technology Research of Foundation Project of Henan Province Department of Science; Projects (1401037A) supported by Natural Science and Technology Research of Foundation Project of Luoyang Department ; Projects (2014M561324) supported by Postdoctoral Science Foundation of China; the science and technology research project of education department of Henan Province (14A510009), the funding scheme for youth teacher of Henan Province (2012GGJS-191);

\section{References}

[1] C. Schuragers, V. Tsiatsis, S. Ganeriwal and Srivastava, "Optimizing sensor networks in the energylatency-density design space, IEEE Trans. Mobile Computer, vol. 12,no. 1, (2012), pp. 1201-1216.

[2] Z. Zaohong, "Analysis of Influencing Factors of Blackboard Teching Effect Based on Ism Model", IIETA International Information and Engineering Technology Association, vol. 12, no. 1, (2014), pp. 916.

[3] X. Luxin, "Research on The Optimization of Enrollment Data Resources Based on Cloud Computing Platform", IIETA International Information and Engineering Tchnology Association, vol. 12, no. 1, (2015), pp. 1-16.

[4] D. Kurt and M. Milos, "WSN configuration-part II : Adaptive coverage for decentralized algorithms", IEEE Trans. Industrial Informatics vol. 20, no. 9, (2012), pp. 1728-1739.

[5] W. Li and W. Zhang, "Coverage analysis and active scheme of WSNs", The Institution of Engineering and Technology, vol. 24, no. 2, (2012), pp. 86-101.

[6] S. Zeyu, W. Weiguo, W. Huanzhao, C. Heng and X. Xingfei, "A novel coverage algorithm based on event-probability-driven mechanism in wireless sensor network", EURSIP Journal on Wireless Communications and Networking, vol. 27, no. 11, (2014), pp. 1-15.

[7] M. Tapiwa, O. Chiwewe, P. Gerhard, and Y. Hancke, "A distributed topology control technique for low interference and energy efficiency in WSN”, IEEE Trans. Industrial Informatics, vol. 8, no. 12, (2012), pp. 11-23.

[8] C. S. Tzong, S. J. Shing, C. T. Yu, "MLPA-conservation mechanism in WSN environments", EURASIP Journal on Wireless Communications and Networking, vol. 25, no. 1, (2012), pp. 1-10.

[9] J. Wang and N. Zhang, "Efficient point coverage in wireless sensor network", Journal of Combinatorial Optimization, vol. 11, no. 10, (2006), pp. 291-302.

[10] A. Capone, M. Cesana, D. Donno and I. Filippini, "Deploying multiple interconnected gateways in heterogeneous wireless sensor networks: an optimization approach", Computer Communications, vol. 33, no. 10, (2010), pp. 1151-1163.

[11] T. Shu and M. Krunz, "Coverage-time optimization for clustered wireless sensor networks: a powerbalancing approach", IEEE/ACM Transactions on Networks, vol. 18, no. 2, (2010), pp. 202-213.

[12] R. Hamid, Y. Karkvandi, R. Efraim and Y. Orly, "Effective Lifetime-Aware routing in WSNs", IEEE Sensors Journal, vol. 11, no. 12, (2011), pp. 3359-3371.

[13] S. Zairi, B. Zouari and E. Niel, "Nodes self-scheduling approach for maximizing WSN lifetime based on remaining energy", The Institution of Engineering and Technology, vol. 2, no. 1, (2012), pp. 52-63.

[14] T. Xueyan and X. Jianliang, "Optimizing lifetime for continuous data aggregation with precision guarantees in WSN", IEEE/ACM Trans. Networking, vol. 16, no. 7, (2008), pp. 904-916.

[15] H. Chihfan and L. Mingyan, "Randomly Duty-cycled WSNs: dynamics of coverage", IEEE Trans. Wireless Communications, vol. 5, no. 11, (2006), pp. 3182-3191.

[16] B. Wang, C. Kee, S. Vikram and W. Wei, "Information coverage in randomly deployed WSN", IEEE Trans. Wireless Communications, vol. 6, no. 12, (2007), pp. 2294-2302.

[17] S. Zeyu, W. Weiguo, W. Huanzhao, C. Heng and W. Wei, "An Optimization Strategy Coverage Control Algorithm for WSN", International Journal of Distributed Sensor Networks, vol. 23, no. 1, (2014), pp. 1-17. 
[18] X. Xingfei, W. Guojun and L. Jie, "Polytype target Coverage Scheme for Heterogeneous Wireless Sensor Networks Using Linear Programming", Wireless Communications and Mobile Computing, vol. 29, no. 10, (2014), pp. 1397-1409.

[19] S. Slijepcevic and M. Potkonjak, "Power Efficient Organization of WSN", IEEE International Journal of Communications, vol. 16, no. 6, (2013), pp. 472-483.

[20] P. Berman, G. Calinescu and C. Shah, "Power efficient monitoring management in sensor networks", IEEE Wireless Communication and Networking, vol. 22, no. 11, (2013), pp. 2329-2339.

[21] D. Tian and N. D. Georganas, "A Node scheduling scheme for energy conservation in large WSNs", Wireless Communications and Mobile Computing, vol. 28, no. 12, (2013), pp. 1271-1283.

[22] Z. Abrams, A. Goel and S. Plotkin, "Set K-Cover algorithms for energy efficient monitoring in WSN", International Journal of Sensor Networks, vol. 16, no. 3, (2010), pp. 424-434.

[23] S. Zeyu, W. Huanzhao, W. Weiguo and X. Xiaofeng, "ECAPM: An Enhanced Coverage Algorithm in Wireless Sensor Network Based on Probability Model", International Journal of Distributed Sensor Networks, vol. 24, no. 1, (2015), pp. 1-16.

[24] X. Yang, C. Hui, W. Kui, S. Bo., Z. Ying, S. Xinyu and L. Chong, "Coverage and detection of a randomized scheduling algorithm in WSNs”, IEEE Trans. Computers, vol. 59,no. 4, (2010), pp. 507-519.

[25] Z. Honghai and C. H. Jennifer, "Maintaining sensing coverage and connectivity in large sensor network", Ad Hoc\& Sensor Wireless Network, vol. 11, no. 2, (2005), pp. 89-96.

\section{Authors}
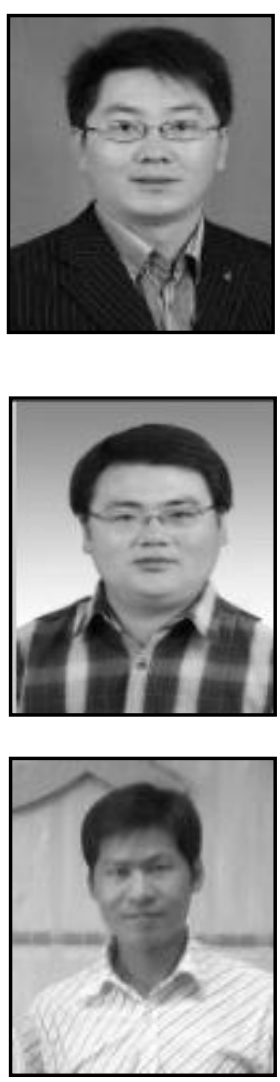

Zeyu Sun, he was born in 1977 in Changchun city, Jilin province, in 2010 graduated from lanzhou university, Master of Science; xi 'an Jiaotong university study for $\mathrm{PhD}$ at present. $\mathrm{He}$ is a lecturer in Luoyang institute of technology of computer and information engineering, is also a member of China computer society. The main research interest is in wireless sensor networks, parallel computing and Internet of things.

Yang Tao, he was born in 1982 in Luoyang City, Henan Province. In 2009, graduated from Lanzhou University, Master of Science, he is the Luoyang Institute of Computer and Information Engineering lecturer; the main research interests are distributed computing, and network information security.

Xiaoming Dong, he is currently the associate Professor of Anqing teachers college, China. University studies: received his B.Sc. in Electrical Engineering and Automation from University of Beijing science and technology in China. He received his M.Sc. from Beijing science and Technology University in China. He received his $\mathrm{PhD}$ from Institute of Automation, Chinese Academy of Sciences Scientific. His research interest fields include machine vision, pattern recognition, wireless sensor networks. 